\title{
Altruism and the Statistical Value of Human Life for Policy and Regulation
}

\author{
Mark C. Long ${ }^{1}$
}

Accepted: 10 August 2021 / Published online: 1 October 2021

(c) The Author(s), under exclusive licence to Springer Nature B.V. 2021

\begin{abstract}
Policymakers use estimates of the Value of a Statistical Life (VSL) in performing regulatory reviews to assess the benefits and costs of policies that affect mortality. This paper empirically estimates an adjustment to conventional VSL measures to account for altruistic sentiments and concludes that conventional VSL measures are underestimated by a factor of two to three, suggesting we are greatly undervaluing life in regulatory reviews. This conclusion is attained by estimating the VSL multiplier suggested by Jones-Lee (Econ $\mathbf{J}$ 102(410):80-90, 1992), which hinges on assessing whether individuals are more health altruistic than wealth altruistic. The estimated VSL multiplier is greatest for policies that affect children's mortality, as health altruism is dominant regarding children.
\end{abstract}

Keywords Benefit-cost analysis · Value of a statistical life $\cdot$ Altruism

\section{Introduction}

The COVID-19 pandemic has brought the tradeoff between money and health into stark clarity. The World Health Organization estimated that the pandemic caused three million deaths worldwide in 2020 (WHO, 2020) and the United States passed 600,000 COVD-19 deaths in June 2021 (Johns Hopkins University, 2021). To respond to this threat, the public has been asked daunting questions about their willingness to sacrifice their economic wellbeing for the sake of their health and the lives of others. The level and types of altruism are key to public decisions. The extent to which individuals are altruistic towards others' health and others' wealth have implications for how much governments should be willing to tax citizens to reduce the probability of death and increase longevity.

U.S. Presidential Executive Order 12,866 requires agencies to conduct a formal benefit-cost analysis when their "proposed or final rule ... may result in aggregate expenditure ... of $\$ 100,000,000$ or more in any one year..." (White House, 2003, p. 43), and most agencies place the "Value of a Statistical Life" (VSL) at around \$10 million (Kniesner \& Viscusi, 2019; Viscusi \& Masterman, 2017). These VSL estimates are derived from revealed

Mark C. Long

marklong@uw.edu

1 Evans School of Public Policy and Governance, University of Washington, 1100 NE Campus Parkway, Seattle, WA 98105, USA 
preference studies that use market transactions which document the amount of money a person is paid to accept some small amount of risk of death or the person's willingness to pay to reduce their risk of death (Mrozek \& Taylor, 2002; Viscusi \& Aldy, 2003) and/ or stated preference contingent valuation studies that ask survey respondents how much money they would be willing to pay for a decrease in the probability of death (Krupnick, 2007; OECD, 2012). Scholars have found variation in VSL based on the age of the person and parents having higher willingness to pay for risk reductions for their children relative to themselves (Carlin \& Sandy, 1991; Liu et al., 2000; Jenkins, Owen, and Wiggins, 2001; Blomquist, 2004; Dickie \& Messman, 2004; Kim, 2004; Aldy \& Viscusi, 2007; Birchenall \& Soares, 2009). Yet, U.S. agencies have not adopted age adjustments given uncertainties in the age-VSL profile as well as political considerations (Graham, 2003; Cropper et al., 2007; NAS, 2008).

Even if regulators sufficiently estimate this age-VSL profile for the valuation of a person's life to themselves, we have the challenge that these valuations may not completely capture the full value of life to all affected parties. If the individual making a market transaction doesn't consider the "the pain and suffering of friends and relatives some of whom may be economically dependent" (Gramlich, 1990, p. 68), then standard benefit-cost analysis will potentially undervalue the person's life. ${ }^{1}$

However, there is no adjustment needed to account for altruism if that altruism is "pure" in the sense that individuals care about the utility of others rather than primarily care about others' probability of survival (Bergstrom, 1982). The intuition for this result is as follows: if person A cares about person B's probability of survival and wealth (net of taxation) in the same proportion as person B cares about their own wealth and own probability of survival, then person A would not be willing to pay more, via a tax levied on person $\mathrm{B}$, for a public safety program than person B is willing to tax himself.

In the case of impure altruism, Jones-Lee (1992) derives the following formula assuming altruism exists solely within families. The value of life that should be used in benefit-cost analysis is equal to the population mean of:

$$
\mathrm{VSL}=m_{i i}\left[\frac{1+\left(x_{k}-1\right) \frac{m_{k i}}{m_{i i}}}{1+\left(x_{k}-1\right) n_{k}}\right]
$$

where $m_{i i}=$ marginal rate of substitution of own wealth for own probability of survival, $m_{k i}=$ marginal rate of substitution of own wealth for a family member's probability of survival, $n_{k}=$ marginal rate of substitution of own wealth for a family member's wealth, and $x_{k}=$ number of family members. Note that if people are pure altruists (i.e., $m_{k i} / m_{i i}=n_{k}$ ) then the expression above reduces down to $m_{i i}$, which is the value used in traditional benefit-costs analysis (if one assumes a constant value of own life across the population: $m_{i i}=m_{j j}=m$ ). If people are more "health-focused" altruists (i.e., $m_{k i} / m_{i i}>n_{k}$ ), then the value in brackets is greater than one, implying that traditional benefit-cost analysis has undervalued life as a result of ignoring altruism. Finally, if people are more

\footnotetext{
${ }^{1}$ Consideration of altruistic sentiments in VSL connects to a broader literature on the intersection of social psychology and non-market transactions. This intersection has been most thoroughly considered in the context of environmental goods (e.g., Jacquemet, James, Luchini, and Shogren (2011) and Neilson and Wichmann (2014)).
} 
"wealth-focused" altruists (i.e., $m_{k i} / m_{i i}<n_{k}$ ), then the value in brackets is less than one, implying that traditional benefit-cost analysis has overvalued life.

Using the more general assumption that individual $i$ can have health and wealth altruism for any other individual $j$, the value of life that should be used in benefit-cost analysis is given by the following (see the supplementary materials, pp. 1-5, for the mathematical derivation):

$$
\mathrm{VSL}=m_{i i}\left[\left(\left(N^{\prime}\right)^{-1} \times V\right)^{\prime} \times M \times D\right]
$$

where $m_{i i}$ is again defined as the marginal rate of substitution of own wealth for own probability of survival, $N$ reflects wealth altruism as an $n \times n$ matrix whose elements, $n_{j i}$, reflect the marginal willingness of person $i$ to forgo own wealth for a gain in person $j$ 's wealth; $V$ is an $n \times 1$ column vector of ones; $M$ reflects health altruism as an $n \times n$ matrix whose elements, $m_{j i}$, reflect the marginal willingness of person $i$ to forgo own probability of survival for a gain in person $j$ 's probability of survival; and $D$ is an $n \times 1$ matrix whose elements reflect the effect of the public project on person $i$ 's probability of survival.

Matrix $D$ will depend on the policy under consideration. Policies that mostly affect older or younger individuals (e.g., investments in cancer research or requirements for bicycle helmets) will have different altruism multipliers if the relative balance between health and wealth altruism varies by the age of the people under consideration. That is, if citizens are more health altruistic towards children and more wealth altruistic towards the elderly (or vice-versa), then policies that affect children's health would have a bigger (smaller) multiplier than policies that effect the elderly.

To date, no one has attempted to estimate this multiplier and there are several challenges in doing so. Jones-Lee noted that there was no existing data on the joint distribution of $m_{k i} / m_{i i}$ and $n_{k}$. Subsequent studies contain evidence that individuals are more healthfocused than wealth-focused altruists (Andersson \& Lindberg, 2009; Hurley \& Mentzakis, 2013; Jacobsson et al., 2007; Long \& Krause, 2017), which would suggest that the multiplier is greater than one. Yet, these studies do not produce a VSL-multiplier estimate. This paper provides the first empirical estimate of this multiplier.

\section{Methodological Overview}

Direct computation of Eq. 2 (i.e., VSL $\left.=m_{i i}\left[\left(\left(N^{\prime}\right)^{-1} \times V\right)^{\prime} \times M \times D\right]\right)$ is impossible. If we give all persons in the world equal standing in the benefit-cost analysis, then the number of elements in the $M$ and $N$ matrices would be equal to the world's population (i.e., $7+$ billion) squared. To fill in these matrices, we would need to know how each person $i$ values the wealth and health of each person $j$. Specifically, we would need to know how much money person $i$ would be willing to forgo in exchange for person $j$ receiving one more dollar and how much person $i$ would be willing to increase their own risk of death for a small reduction in the risk of death of person $j$. 
To feasibly estimate the multiplier, I created a stated-preference survey to elicit the respondent's wealth and health altruism. ${ }^{2}$ I use the results of that survey to simulate a world with around 1,400 persons, where this number corresponds to the 1,144 respondents in the survey plus their 231-280 simulated minor children. For these 1,400 people, I construct simulated bilateral relationships, including whether the pair are together in immediate or extended families, close friends, co-workers, acquaintances, or domestic or foreign strangers. I utilize data collected from these respondents on the number and age distribution of their real-life relationships to ground the simulated world. I then fill-in the matrices using estimates of altruistic preferences. I evaluate the sensitivity of the resulting estimated multiplier to (1) whether close friends, co-workers, and acquaintances are spread randomly across the matrices (a "loosely" connected world) or hold tightly to a diagonal across these matrices, (2) whether strangers are mostly domestic or foreign, (3) whether the public policy affecting public safety has uniform effects across the population or affects members of particular age groups, (4) the order in which persons are entered into the matrices, and (5) uncertainty in the parameters which are used to estimate wealth and health altruism.

It is undoubtedly the case that the resulting estimates will not perfectly match the "true" multiplier that would be obtained if we could know the wealth and health altruism of every person on Earth towards every other person on Earth. Rather, the goal of this exercise is to evaluate whether this multiplier appears to be substantially different than 1 , and how it might be sensitive to various assumptions. The next sections discuss the methodology in greater detail.

\section{Construction of the Matrices Step 1: Establishing Social Relations}

For each respondent to the survey described below, I define all relationships between person $i$ and person $j$ in a manner that tries to mimic the survey respondents' stated numbers and ages of family members, friends, co-workers, and acquaintances. I begin by constructing pseudo-families.

For respondent $i$ who has a living parent, I consider whether respondent $j$ is similar to their living parents. I generate a "score" for each potential parent and identify which other respondent has the highest score and is thus the best "parent" for respondent $i$. Respondent $j$ gets a higher score if: $j$ has an adult child, $j$ has a spouse and $i$ has two or more living parents, $j$ has no spouse and $i$ has one living parent, $j$ 's children are close in number to $i$ 's siblings, $j$ 's age is similar to $i$ 's parents' ages, and $i$ 's age is similar to $j$ 's children's ages. I conduct similar exercises to score each person $j$ for their similarity to $i$ 's adult siblings and spouse.

The next set of steps are repeated 100 times and the median multiplier is reported from among these iterations. In each iteration, I randomly sort the respondents. ${ }^{3}$ I begin with respondent $i=1$ and build this respondent's birth and marital families identifying those

\footnotetext{
${ }^{2}$ The survey was fielded in the summer of 2015 by Knowledge Networks to 1,144 members of their KnowledgePanel®, which is an online panel based on a representative sample of the full U.S. non-institutionalized population (GfK, 2013). Appendix C contains additional information on the survey, including descriptive statistics (Appendix Table 1). The resulting dataset is available here: https://www.openicpsr.org/ openicpsr/project/100788/version/V1/view.

${ }^{3}$ Data construction is conducted using Stata (2019). The seed was set at 3 as the beginning point for the pseudo-random number generation. For replication purposes, all coding associated with the paper is available from the author.
} 


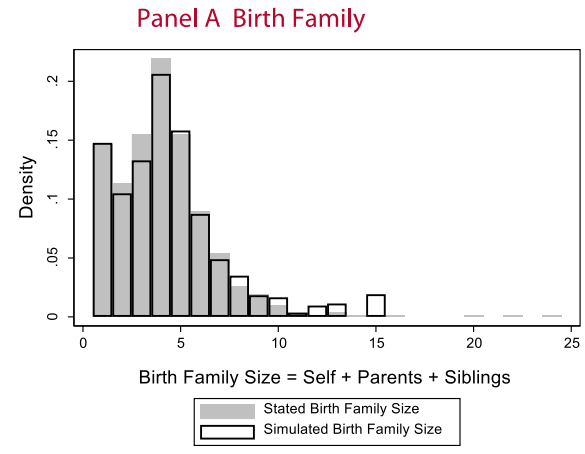

Panel C Extended Family

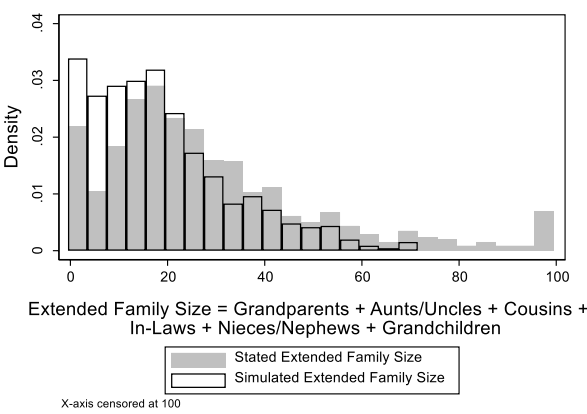

Panel B Marital Family

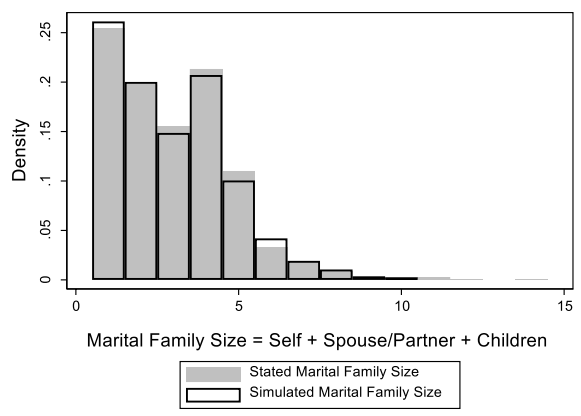

Panel D Extended Family Excluding Cousins

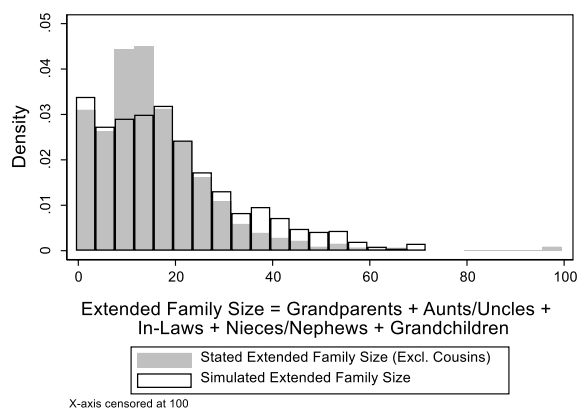

Fig. 1 Distribution of family sizes, stated versus simulated

persons $j$ with the best matches. I add $i$ 's minor children in accordance with $i$ 's survey responses and establish simulated relationships within members of $i$ 's birth and marital families (i.e., parent to child and sibling to sibling relationships). I proceed with persons $i=2$ to 1,144 while not overwriting previously established bilateral relationships. Once all 1,144 respondents have their birth and marital families constructed, I assign all resulting extended family relationships (i.e., constructing grandparent to grandchild, aunt/uncle to nephew/niece, mother/father-in-law to son/daughter-in-law relationships, and cousins). Figure 1, which contains the relationships from the first of the 100 constructed matrices, shows that this process yields a simulated community whose numbers of simulated immediate familial relationships match well with respondents' reported family sizes, but somewhat underestimates respondents' number of reported extended family members, particularly cousins.

Next, I sort the observations so that they most tightly cluster into family units. The reason that I do this sorting is (a) it seems natural that families would live close to each other in social space and (b) sorting matters for the final estimates of the multipliers. Once completed, the 1,400 persons in the matrix are aligned such that $i$ and $j$ are unlikely to have family relations if their index numbers are far apart.

To establish close friend, co-worker, and acquaintance relationships, I take two polar extreme positions. First, I use the "loosest" possible approach, which randomly selects an $i$ and a $j$ and establishes the relationship between the two if $i$ and $j$ do not have an already established relationship and $i$ and $j$ are each seeking a relation with a person of the other's 
Panel A: Tight Non-Family Relations

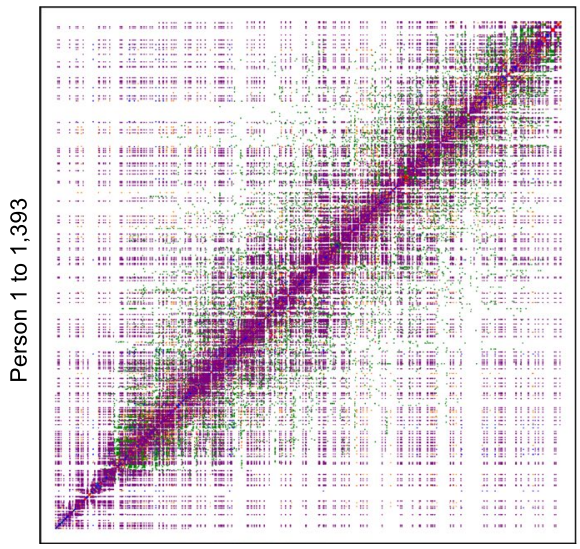

Person 1 to 1,393
Panel B: Loose Non-Family Relations

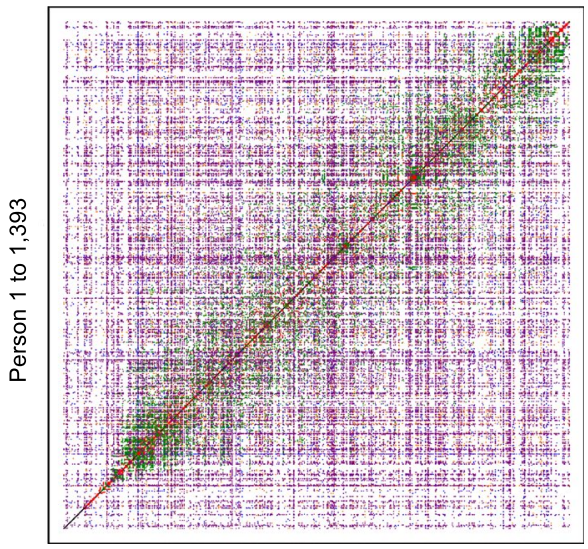

Person 1 to 1,393

\section{- Self \\ - Extended Family \\ Immediate Family \\ Co-Worker \\ - Close Friend \\ Acquaintance}

Fig. 2 Illustration of tight and loose non-family social relations

type. For example, if individual $i$ is 25 years old and reports in the survey having a close friend age $30-39$, and individual $j$ is 34 years old and reports having a close friend age 20-29, and $i$ and $j$ have not as yet been assigned a relationship, then they will be established as being close friends. This "loose" approach (which randomly selects $i$ and $j$ to identify possible relationships) yields a society that has the fewest possible degrees of separation between pairs of individuals. Second, I use the "tightest" possible approach, which seeks out persons $i$ and $j$ that are closest to the diagonal of the matrix. This tight approach yields a matrix that has the most degrees of separation between pairs of individuals.

Figure 2 shows these two matrices for the first of the 100 matrix constructions. These matrices contain nearly 2 million potential relationships (precisely, $1,413 \times 1,413=1,996,569$, which includes 1,144 respondents and their 259 simulated minor children). Familial relationships are identical in the two panels. This fact can most easily be seen by the clusters of red and green cells in the lower-left and upper-right-hand corners, which reflects large families with large numbers of extended family members. The two panels differ in the assumed spatial distributions of close friends, co-workers, and acquaintances. We can think of the "tight" relations shown in panel A as reflective of a large village where individuals do not travel far away from their clans, whereas panel B reflects a dynamic and freely flowing community where relationships happen randomly. If the resulting multipliers vary for these polar extremes, then it would suggest that context and local conditions are important to the size of the multiplier. As shown below, however, the multipliers are only modestly different based on the tightness of social relations.

Most of the area in Panels A and B in Fig. 2 is not colored as most of the individuals are strangers to each other. Thus, even in this relatively small, simulated world, most people are strangers and thus altruistic sentiment towards strangers may play an important role in 
the multiplier. In the estimates below, the stranger relationships are alternately assumed to be U.S. strangers and strangers from other countries. Additionally, I compute matrices where there are no family, friends, co-workers, or acquaintances, and rather all off-diagonal elements are either U.S. or foreign strangers. These latter matrices will produce results that are closer to accurate when considering policy from a U.S. national or whole world perspective where almost everyone is a stranger.

In total, 600 matrices of relationships are produced: 100 random sortings of the 1,144 survey respondents $\times 6$ types (i.e., tight relations with U.S. strangers, tight relations with foreign strangers, loose relations with U.S. strangers, loose relations with foreign strangers, everyone is a U.S. stranger, or everyone is a foreign stranger).

\section{Construction of the Matrices Step 2: Person i's Altruism Towards Person $j$}

Health altruism $\left(\frac{m_{j i}}{m_{i i}}=\frac{\partial \pi_{j}}{\partial \pi_{i}}\right)$ is the most difficult term to estimate since there are not realworld contexts where the typical person is "revealed" to show their valuation of own health relative to another's health (i.e., the marginal willingness of person $i$ to exchange a slight increase in the probability of their own death for a slight decrease in the probability of person $j$ 's death). As such, at this time, it is only feasible to use survey-based stated preferences to estimate health altruism.

Wealth altruism $\left(\frac{n_{j i}}{n_{i i}}=\frac{\partial w_{j}}{\partial w_{i}}\right)$ does have real-world behavior (e.g., charitable donations) and experiments (e.g., "dictator games") from which to estimate this wealth altruism. In such games, one study participant (the "dictator") is given the opportunity to divide a fixed amount of money between themselves and another study participant. In the average study, dictators are found to give away $28 \%$ of the money to the other participant (Engel, 2011). ${ }^{4}$ Yet for the purposes of estimating the multiplier, I again use stated preferences.

Survey questions were constructed so as to elicit estimates of health and wealth altruism in a parallel fashion such that if the results yield biased estimates of altruism, it is hoped that the biases are of comparable magnitudes such that they cancel out. For example, returning to Eq. 2, if the estimates included in the $M$ and $N$ matrices are biased upwards (or downwards) by the same percentage amount, these biases will cancel.

Survey respondents were asked to choose between two mutually exclusive options. ${ }^{5}$ To elicit levels of health altruism, respondents were told to "imagine that a company decided to give out special medical products and/or safety inventions that could lower a person's chance of dying during the next 10 years." The respondents were asked to "decide if the company should either give you a given number of medical products or safety inventions

\footnotetext{
4 There are other experimental methods that elicit wealth altruism such as "trust games" (where the first participant may send a portion of money to another participant, the money is increased by some multiple, and then the second participant may return a portion of the inflated sum back to the first participant) and "public goods games" (where all participants may contribute a portion of their money to a common pot, the pot is increased by some multiple, and then all share equally in the pot) (Levitt and List, 2007). Dictator games are the closest experimental method to the stated preference survey method used in this paper as the respondent is asked how much of a fixed stock to share with another person.

5 Note that language in this section parallels that in Authors (Blinded), which uses the same survey to more fully describe the correlates of altruistic responses. We also describe the result of a second survey that framed the choice as a dictator game, and found similar results (i.e., more health than wealth altruism).
} 
or give some other person a given number of medical products or safety inventions." The respondents were told the age range of the other person (for example, 40-49 year old), and their relationship to the respondent (for example, "acquaintance"). The respondent was only asked questions about a relationship type that the person reported having. For example, the respondent was only given a question about health altruism towards an immediate family member age 10-17 if the respondent reported having such a relationship in earlier questions. In each question, the respondent was asked to select whether they would like the products/inventions to go to themself or to the other person.

For example, the respondent was asked to "select the option that you most prefer" and told to remember that "each product/invention increases the recipient's chance of surviving 10 years by 1 chance in 10,000" and given the following graphic:

\begin{tabular}{|l|l|}
\hline$\square$ & $\begin{array}{l}\text { Option 1: } \\
\text { The company gives } 8 \text { medical products or safety inventions to } \\
\text { you (and gives nothing to the other person). }\end{array}$ \\
\hline$\square$ & $\begin{array}{l}\text { Option } 2: \\
\text { The company gives } 8 \text { medical products or safety inventions to a } \\
40-49 \text { year old acquaintance (and gives nothing to you). }\end{array}$ \\
\hline
\end{tabular}

Option's 1 and 2 form a discrete choice binary variable. If the respondent selected Option 1 when faced with the above choice, reflecting more regard for the health of herself than the other person, then the bold, underlined numbers were changed to 4 versus 8 and the question was asked again so to assess how far the regard for self over the other person goes. If the respondent continued to select Option 1, the respondent was given progressively less generous scenarios ( 2 versus 8,1 versus 8 , and finally 1 versus 15 ). Otherwise, if the respondent switched to Option 2 when faced with 4 versus 8 , they were then given a new scenario where the ratio was increased to 6 versus 8 .

The object is to find the point at which the respondent switched from Option 1 to Option 2. This switching point reflects the respondent's marginal willingness to exchange a slight increase in the probability of their own death for a slight decrease in the probability of the other person's death.

For example, if the respondent switched from Option 1 to Option 2 between 6 v. 8 and 5 v. 8, then we should conclude that the respondent was willing to decrease their survival probability by more than 5 chances in 10,000 (but less than 6 chances in 10,000) to increase the other person's survival probability by 8 chances in 10,000 . Thus, the marginal rate of substitution (MRS) for this person lies in the interval between $\frac{5}{8}$ and $\frac{6}{8}$. The series of questions allows us to identify that the MRS lies in one of 17 intervals: below $\frac{1}{15}$; between the following: $\frac{1}{15}, \frac{1}{8}, \frac{2}{8}, \frac{3}{8}, \frac{4}{8}, \frac{5}{8}, \frac{6}{8}, \frac{7}{8}, \frac{8}{8}, \frac{9}{7}, \frac{10}{6}, \frac{11}{5}, \frac{12}{4}, \frac{13}{3}, \frac{14}{2}$, and $\frac{15}{1}$; or above $\frac{15}{1}$. Given that the MRS is categorical and ordered with an ordinal scale, an ordered-probit regression is used, as described below. If the person always selected Option 2 (even when offered 15 to self versus 1 to the other person), then they were asked a question to identify whether they reject the scenario (e.g., they do not want the medical products/safety inventions even if they were given for free). Respondents who rejected the scenario were dropped from the analytical sample used in the ordered probit regressions. ${ }^{6}$

\footnotetext{
6 The sample size for the regression is 10,834 (i.e., 1,144 participants $\times(5$ health altruism scenarios presented per participant +5 wealth scenarios presented per participant) -606 scenarios that were dropped due to failure to answer the question or scenario rejection).
} 
The wealth altruism questions were constructed in a parallel fashion, where the respondent was told to "(i)magine that a company decided to give out scratch-off tickets and that each ticket has one chance in 10,000 of winning \$25,000 from the company." Again, the respondent was asked to choose between Options 1 and 2 (i.e., to select whether she would like the given numbers of scratch-off tickets to go to herself or to the other person). After making the initial choice, the respondent was presented a new choice where the number of scratch-off tickets was adjusted to be either more or less generous to the other person. (These adjustments in the numbers of scratch-off tickets paralleled the adjustment of the numbers of medical products/safety invention). The ratio of the numbers of tickets offered in Option 1 (to self) and Option 2 (the other person) at which the respondent switched from Option 1 to Option 2 yields the respondent's marginal willingness to forgo her own wealth for a gain in the other person's wealth.

The respondent's health/wealth altruism is assumed to be given by the following linear function:

$$
\begin{aligned}
\operatorname{MRS}_{h i j}^{*}= & \left(P_{i j}^{\prime} \beta_{1}+A_{j}^{\prime} \beta_{2}+X_{i}^{\prime} \beta_{3}+F_{i}^{\prime} \beta_{4}\right) H_{h} \\
& +\left(\gamma_{0}+P_{i j}^{\prime} \gamma_{1}+A_{j}^{\prime} \gamma_{2}+X_{i}^{\prime} \gamma_{3}+F_{i}^{\prime} \gamma_{4}\right)\left(1-H_{h}\right)+u_{i}+\varepsilon_{i j},
\end{aligned}
$$

and the observed categorical responses, $y_{h i j}$, are given by:

$$
y_{h i j}=\left\{\begin{array}{c}
1 \quad \text { if } M R S_{h i j}^{*} \leq \tau_{1} \\
2 \text { if } \tau_{1}<M R S_{h i j}^{*} \leq \tau_{2} \\
\vdots \\
17 \text { if } \tau_{16}<M R S_{h i j}^{*}
\end{array}\right.
$$

where $M R S_{h i j}^{*}$ is the latent marginal rate of substitution between person $i$ 's own health (wealth) with respect to person $j$ 's health (wealth); $P_{i j}$ reflects a vector of 6 categories of social proximity between $i$ and $j$ (with foreign stranger as the base category); $A_{j}$ is a vector of 9 age categories for hypothetical person $j$ (with age 80 and older omitted as the base category); $X_{i}$ is a vector of characteristics of person $i$ (including an indicator for female; age and age-squared; and a vector of categorical measures of distance between birth location and current state of residence); $F_{i}$ is a vector of framing effects (health v. wealth questions asked first; whether the "other person" was shown as Option 1 or 2; the interaction of these two; and whether the respondent was initially asked about an equal allocation, or a less or more generous allocation); $H_{h}$ is an indicator variable that equals one (zero) if the person is answering a question about health altruism and equals zero if the person is answering a question about wealth altruism ${ }^{7} ; u_{i}$ is a respondent-specific random intercept which is assumed to be distributed $N\left(0, s^{2}\right)$ with $s^{2}$ estimated in the course of the regression; $\varepsilon_{i j}$ is assumed to be distributed $N(0,1)$ and independent of $u_{i}$; and $\tau_{k}$ are estimated threshold values.

This specification is estimated using a mixed effects ordered probit with respondentspecific random intercepts, yielding estimates of the $\beta, \gamma$, and $\tau$ coefficients and $s^{2}$ using the specification from Long and Krause (2017). Results of this regression are found in

\footnotetext{
7 Note that the $\beta$ coefficients are identified from observations where $H_{h}=1$ and the $\gamma$ coefficients are identified from observations where $H_{h}=0$.
} 
Appendix Table 2. ${ }^{8}$ To evaluate the extent to which the estimated multiplier is affected by parameter uncertainty, I take 10 bootstrapped samples, estimate the parameters for each sample, and discuss below the impacts of this uncertainty. ${ }^{9}$

Next, I fill in the elements of the $M$ and $N$ matrices as follows. First, I randomly draw one value of $u_{i}$ from a $N\left(0, \widehat{s^{2}}\right)$ distribution - think of this value as the person's base-level of general altruism. Ten values from this distribution for each person are drawn and I evaluate the extent to which this individual-specific base altruism uncertainty affects the estimated multiplier. For health altruism, this individual-specific base altruism is added to $P_{i j}^{\prime} \widehat{\beta}_{1}+A_{j}^{\prime} \widehat{\beta}_{2}+X_{i}^{\prime} \hat{\beta}_{3}+F_{i}^{\prime} \hat{\beta}_{4}$ to estimate $\widehat{y_{1 i j}}$, which is then transformed into an entry of the $M$ matrix using the following conversion: $\frac{1}{20}$ for $\widehat{y_{1 i j}}=1$; the range's midpoint MRS (e.g., $\frac{3}{16}$ for the range $\frac{1}{8}$ to $\frac{2}{8}$ ) for $1<\widehat{y_{1 i j}}<17$; or 20 if $\widehat{y_{1 i j}}=17$. The same procedure is done to fill in the $N$ matrices, yet with $u_{i}$ added to $\hat{\gamma_{0}}+P_{i j}^{\prime} \hat{\gamma_{1}}+A_{j}^{\prime} \hat{\gamma_{2}}+X_{i}^{\prime} \hat{\gamma_{3}}+F_{i}^{\prime} \hat{\gamma_{4}}$.

The VSL multiplier, shown in square brackets in Eq. 2, can now be computed given an assumed distribution of the effects of the public project on each individual's probability of survival. First, I assume that each person receives an equal-sized increase in their survival probability and the public policy saves one life in expectation (e.g., that each element of the $D$ matrix equals $\sim 1 / 1,400$ ). Then, I assume that the public policy only affects the survival probability of those in one particular age group (e.g., only affecting those age 0-4) and that each member of this age group has their probability of survival raised by $1 / K$, where $K$ is the number of members of that age group. With these simulations, I show the extent of the change in the multiplier under various conditions.

\section{Results}

Table 1 presents the key results, showing the median VSL multiplier assuming a public policy that affects all age groups equally. The median VSL multiplier ranges from 1.88 to 3.24 with relatively small standard errors, ranging from 0.04 to 0.07 . I can reject that the VSL multiplier equals 1 with high confidence. Generally, the multiplier is higher if strangers are assumed to come from the U.S. rather than abroad or if all non-diagonal elements are assumed to be strangers from either the U.S. or outside the U.S.

When the matrices include non-strangers (family members, friends, co-workers, and acquaintances) around two-thirds of the variance in the estimate of the multiplier is attributable to the order in which the respondents were randomly sorted prior to building social relations. Whereas, when all non-diagonal elements are assumed to be strangers, more than half of the variance comes from uncertainty about parameters or the draw of the individual's base altruism $\left(u_{i}\right)$.

\footnotetext{
8 In describing these regression results, Long and Krause note:

"Controlling for other factors, respondents in both surveys gave significantly more to all relationship types relative to foreign strangers (except for co-workers, where the differences with foreign strangers were mostly insignificant). The most altruism was exhibited towards immediate family members for both surveys and for both health and wealth questions....Health altruism is highest for those age 0-4 and 5-9 in both surveys, while wealth altruism is lower for these age groups, and these differences in treatment for the health and wealth questions are statistically significant.".

This paper amends one line of code from Long and Krause (2017) to better measure distance between place of birth and state of current residence, which has modest effects on the results.

9 These bootstrapped samples were created using the Stata (2019) command "bsample" and were clustered by respondent.
} 


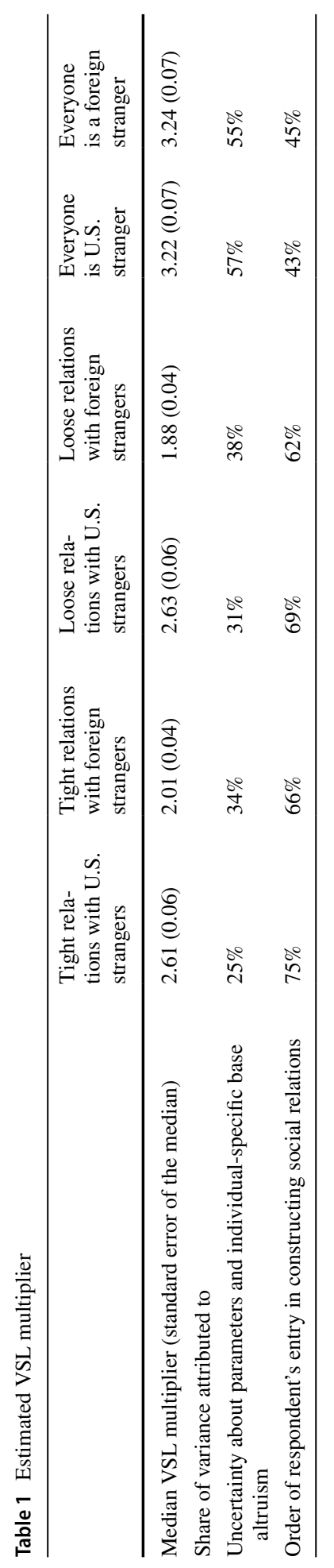




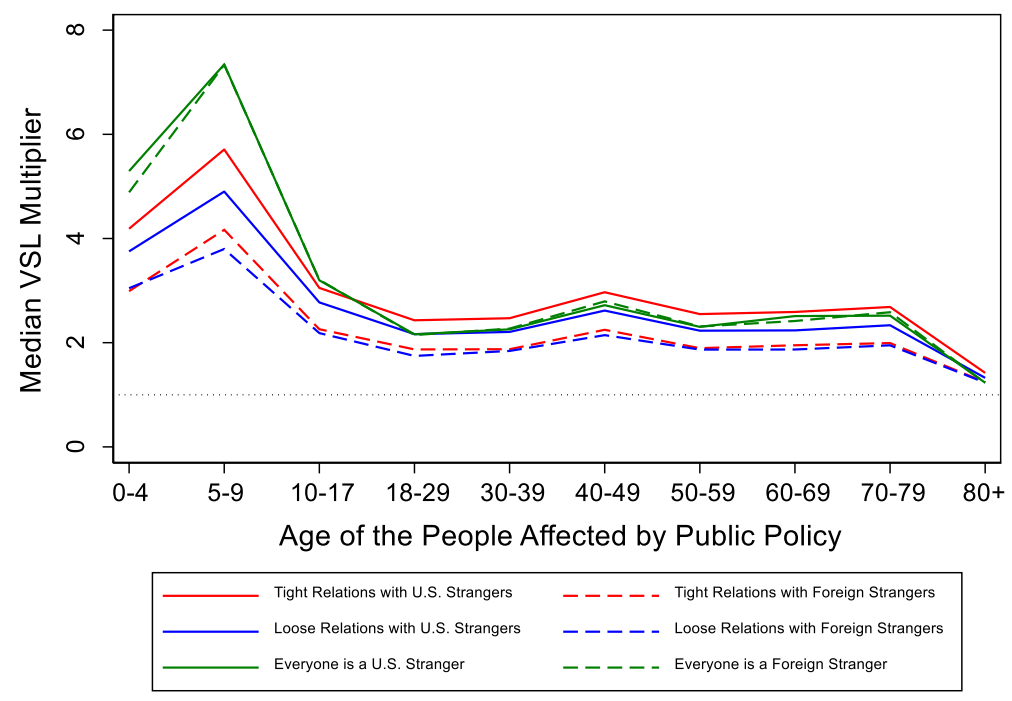

Fig. 3 Estimated VSL multiplier by age of those affected by public policy

Figure 3 shows how this median VSL multiplier varies if the public policy only affects the survival probability of persons from particular age ranges. The median VSL multiplier is much higher for public policies that affect the survival of children under the age of 10, reaching as high as 7.3 for matrices that only consist of U.S. or foreign strangers. This result suggests that altruistic sentiments towards children favor health over wealth altruism and mirror findings showing parents having higher willingness to pay to avoid death for their children relative to themselves. Given this large VSL multiplier for policies affecting children's mortality, policymakers would find more support for decisions such as the 2019 recall of Fisher-Price's Rock'n Play inclined sleeper, which was coordinated with the Consumer Product Safety Commission (Frankel, 2021), and the House Committee on Oversight and Reform's (2020) efforts to urge the National Highway Traffic Safety Administration to improve children's car seat and booster seat safety.

Figure 3 further shows that for individuals aged 10 to 79, the multiplier ranges from 1.75 to 3.21 . While health altruism declines with respect to the age of the other person (Long \& Krause, 2017), I nonetheless find that the median VSL multiplier is greater than one for persons aged 80 and above, ranging from 1.23 to 1.42 .

\section{Discussion}

The presence and nature of altruistic sentiments yields a social Value of a Statistical Life that is substantially and significantly higher than what is used in current U.S. federal policy, which ignores altruistic sentiments and bases VSLs on individuals' valuations of themselves. This result occurs because people report caring more for the health of others than the wealth of others and consequently would be more willing to tax others to pay for public projects that raise public safety than these others would be willing to tax themselves.

If the VSL multiplier that is suggested by this paper were used in benefit-cost analyses and regulatory decisions, it would favor adoption of interventions that save and 
extend lives, particularly policies that affect the mortality of children. However, as policy shifted in the direction of health interventions it is reasonable to believe that the perceived marginal benefit of saving and extending lives would go down. If this occurred, we might see a shift away from altruistic preferences towards health and towards altruistic preferences towards wealth and increasing public desire for expenditure of resources on other public goods (e.g., education, environmental quality, economic development) rather than health. If this were to occur, then the VSL multiplier would diminish overtime endogenously.

These results should, of course, be taken with a grain of salt as they are based on stated preferences for giving, and these may be substantially greater than true, unobserved altruistic preferences. However, given the size of the estimated VSL multiplier, these results should cause public officials to question their methods and academic researchers to seek additional methods to estimate this multiplier. Estimating this multiplier is challenging as there are few contexts in which the public can reveal their preferences and show their willingness to trade their own probability of death for another's probability of death. Future research on this topic should consider alternative methods for eliciting truthful revelation of subjective altruistic sentiments, perhaps using incentive-design such as the approach outlined in Prelec (2004). Yet, even if the stated preferences yield biased estimates of the unobserved altruistic preferences that would be revealed in real-world behavior (if such contexts existed), such results may still be valid if they reveal the public's altruistic aspirations. There is a reasonable argument that the VSL that should be used in regulatory analysis should be based on such aspirations as they correspond to the public's desired valuations.

It should be noted that many people find the notion of placing a value on human life to be distasteful and perhaps immoral (Cameron, 2010). In particular, incorporating variation in the value of life used in benefit-cost analysis as a function of the age of the victims (beneficiaries), or as a function of the social proximity of the victims (beneficiaries) to the persons paying taxes to fund the project seems distasteful to many (Viscusi, 2009). If standard benefit-cost analysis were to adopt such age and social proximity adjustments, the political appeal of benefit-cost analysis as an analytical tool may be weakened (Cropper et al., 2007; NAS, 2008). While it is unclear whether including altruistic sentiments would lessen or enhance public confidence in the validity of benefit-cost analysis, the challenges with estimating such altruism is sure to invite skeptical scrutiny.

The ultimate goal with this research is to produce evidence that would help scholars know whether altruistic sentiments are likely to be important in estimating the VSL. Given the magnitude of the VSL multiplier estimated here, I hope that this research serves as a catalyst for a new literature that re-conceptualizes the VSL from a social point of view.

Supplementary Information The online version contains supplementary material available at https://doi. org/10.1007/s11205-021-02784-7.

Acknowledgements Support for this research came from the National Science Foundation (Award ID 1457913), University of Washington Innovation Award, University of Washington's Research Royalty Fund, and a Eunice Kennedy Shriver National Institute of Child Health and Human Development research infrastructure grant (R24 HD042828) to the Center for Studies in Demography \& Ecology at the University of Washington. Helpful comments were provided by Ann Bostrom, Joe Cook, James Cowan, Crystal Hall, Meg Hargreaves, Ines Jurcevic, Spiro Maroulis, Lisa Robinson, anonymous reviewers, and conference participants at the Society for Benefit-Cost Analysis and Association for Public Policy Analysis \& Management. Excellent research assistance was provided by Nicole Bateman, Grant Blume, Eleanor Krause, Stephanie Leiser, Jordan Rooklyn, Hilary Wething, Jason Williams, and Mariam Zameer. 


\section{References}

Aldy, J. E., \& Viscusi, W. K. (2007). Age differences in the value of statistical life: Revealed preference evidence. Review of Environmental Economics and Policy, 1(2), 241-260.

Andersson, H., \& Lindberg, G. (2009). Benevolence and the value of road safety. Accident Analysis and Prevention, 41(2), 286-293.

Bergstrom, T. C. (1982). When is a man's life worth more than his human capital? In M. W. Jones-Lee (Ed.), The Value of Life and Safety: proceedings of a conference held by the Geneva Association (pp. 3-26). Amsterdam: North Holland.

Birchenall, J. A., \& Soares, R. R. (2009). Altruism, fertility, and the value of children: Health policy evaluation and intergenerational welfare. Journal of Public Economics, 93, 280-295.

Blomquist, G. C. (2004). Self-protection and averting behavior, values of statistical lives, and benefit cost analysis of environmental policy. Review of Economics of the Household, 2(1), 89-110.

Cameron, T. A. (2010). Euthanizing the Value of a Statistical Life. Review of Environmental Economics and Policy, 4(2), 161-178.

Carlin, P. S., \& Sandy, R. (1991). Estimating the implicit value of a young child's life. Southern Economic Journal, 58(1), 186-202.

Cropper, M., et al. (2007). SAB advisory on EPA's issues in valuing mortality risk reduction (EPASAB-08-001). U.S. Environmental Protection Agency Science Advisory Board.

Dickie, M., \& Messman, V. L. (2004). Parental altruism and the value of avoiding acute illness: Are kids worth more than parents? Journal of Environmental Economics and Management, 48(3), 1146-1174.

Engel, C. (2011). Dictator games: A meta study. Experimental Economics, 14(4), 583-610.

Frankel, T. C. (2021). House panel grills executives over popular Fisher-Price infant sleeper tied to more than 90 deaths. Washington Post, June 7.

GfK. (2013). "KnowledgePanel® Design Summary.” Accessed on April 15, 2016 from http://www.knowl edgenetworks.com/knpanel/docs/knowledgepanel(R)-design-summary-description.pdf.

Graham, J. D. (2003, May 30). Benefit-cost methods and lifesaving rules (memorandum to the President's Management Council). Washington DC: Office of Information and Regulatory Affairs, Office of Management and Budget. Accessed on June 16, 2021, https://www.whitehouse.gov/sites/whitehouse.gov/ files/omb/assets/regulatory_matters_pdf/pmc_benefit_cost_memo.pdf.

Gramlich, E. M. (1990). A guide to benefit cost analysis. Prentice-Hall.

House Committee on Oversight and Reform. (2020). Oversight subcommittee seeks briefing from NHTSA on children's car and booster seat safety standards. Press release, Nov. 19. Accessed on June 17, 2021, https://oversight.house.gov/news/press-releases/oversight-subcommittee-seeks-briefing-from-nhtsaon-children-s-car-and-booster

Hurley, J., \& Mentzakis, E. (2013). Health-related externalities: Evidence from a choice experiment. Journal of Health Economics, 32(4), 671-681.

Jacobsson, F., Johannesson, M., \& Borgquist, L. (2007). Is altruism paternalistic? Economic Journal, 117(520), 761-781.

Jacquemet, N., James, A., Luchini, S., \& Shogren, J. (2011). Social psychology and environmental economics: A new look at ex ante corrections of biased preference evaluation. Environmental \& Resource Economics, 48(3), 413-433.

Jenkins, R. R., Owens, N., \& Wiggins, L. B. (2001). Valuing reduced risks to children: The case of bicycle safety helmets. Contemporary Economic Policy, 19(4), 397-408.

Johns Hopkins University. (2021). Coronavirus Research Center. Accessed on June 16, 2021, https://coron avirus.jhu.edu/

Jones-Lee, M. W. (1992). Paternalistic altruism and the value of statistical life. Economic Journal, 102(410), 80-90.

Kim, H. S. (2004). Measuring the value of statistical life in the U.S.A. by means of risk assessment of family automobile purchases. Studies in Regional Science, 34(3), 225-236.

Kniesner, T. J., \& Viscusi, W. K. (2019). The value of a statistical life. Oxford University Press.

Krupnick, A. (2007). Mortality-risk valuation and age: Stated preference evidence. Review of Environmental Economics and Policy, 1(2), 261-282.

Levitt, S. D., \& List, J. A. (2007). What do laboratory experiments measuring social preferences reveal about the real world? Journal of Economic Perspectives, 21(2), 153-174.

Liu, J.-T., Hammitt, J. K., Wang, J.-D., \& Liu, J.-L. (2000). Mother's willingness to pay for her own and her child's health: A contingent valuation survey in Taiwan. Health Economics, 9(4), 319-326.

Long, M. C., \& Krause, E. (2017). Altruism by age and social proximity. PLoS ONE, 12(8), e0180411. https://doi.org/10.1371/journal.pone.0180411 
Mrozek, J. R., \& Taylor, L. O. (2002). What determines the value of life? A meta-analysis. Journal of Policy Analysis and Management, 21, 253-270.

National Academy of Sciences Committee on Estimating Mortality Risk Reduction Benefits from Decreasing Tropospheric Ozone Exposure (NAS). (2008). Estimating mortality risk reduction and economic benefits from controlling ozone air pollution. National Academies Press.

Neilson, W., \& Wichmann, B. (2014). Social networks and non-market valuations. Journal of Environmental Economics and Management, 67(2), 155-170.

Organisation for Economic Co-operation and Development (OECD). (2012). Mortality risk valuation in environment, health and transport policies. Accessed on March 27, 2020 at http://www.oecd.org/env/ tools-evaluation/mortalityriskvaluationinenvironmenthealthandtransportpolicies.htm\#How_to_obtain_ this_publication

Prelec, D. (2004). A Bayesian truth serum for subjective data. Science, 306(5695), 462-466.

StataCorp. (2019). Stata Statistical Software: Release 16. StataCorp LLC.

Viscusi, W. K. (2009). The devaluation of life. Regulation \& Governance, 3, 103-127.

Viscusi, W. K., \& Aldy, J. E. (2003). The value of a statistical life: A critical review of market estimates throughout the world. Journal of Risk and Uncertainty, 27(1), 5-76.

Viscusi, W. K., \& Masterman, C. J. (2017). Income elasticities and global values of a statistical life. Journal of Benefit Cost Analysis, 8(2), 226-250.

White House. (2003). Circular A-4, "Regulatory Analysis". Accessed on June 16, 2021, https://www.white house.gov/sites/whitehouse.gov/files/omb/circulars/A4/a-4.pdf

World Health Organization (WHO). (2020). Global Preparedness Monitoring Board. A World in Disorder. Global Preparedness Monitoring Board Annual Report 2020. Accessed on June 16, 2021, https://apps. who.int/gpmb/assets/annual_report/GPMB_AR_2020_EN.pdf

Publisher's Note Springer Nature remains neutral with regard to jurisdictional claims in published maps and institutional affiliations. 